
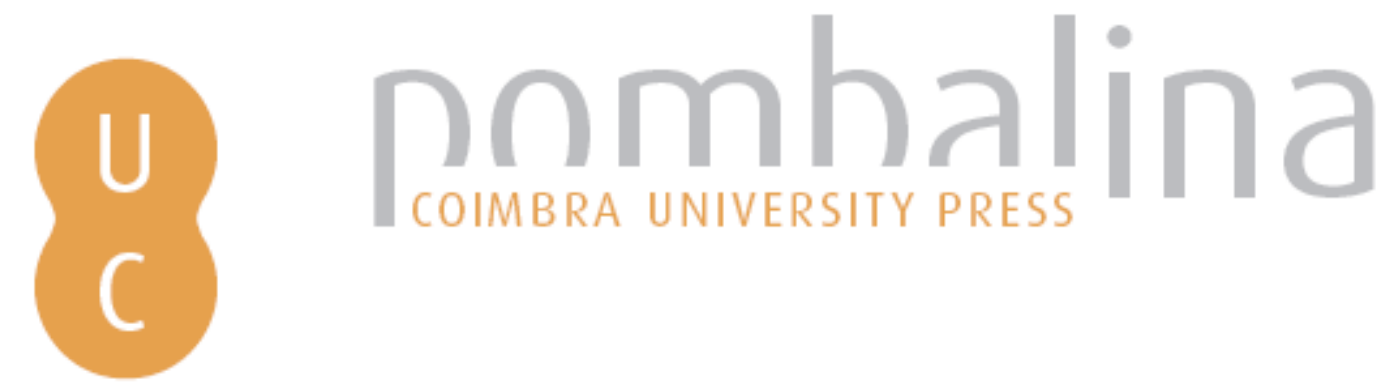

Influencia de los progymnasmata en la composición de los symposia de Plutarco: el caso de El Banquete de los Siete Sabios

Autor(es): $\quad$ Vicente Sánchez, Ana

Publicado por: Imprensa da Universidade de Coimbra; Centro de Estudos Clássicos e

URL

persistente: URI:http://hdl.handle.net/10316.2/31980

DOI: $\quad$ DOl:http://dx.doi.org/10.14195/978-989-8281-17-3_7

Accessed : $\quad$ 26-Apr-2023 04:08:38

A navegação consulta e descarregamento dos títulos inseridos nas Bibliotecas Digitais UC Digitalis, UC Pombalina e UC Impactum, pressupõem a aceitação plena e sem reservas dos Termos e Condições de Uso destas Bibliotecas Digitais, disponíveis em https://digitalis.uc.pt/pt-pt/termos.

Conforme exposto nos referidos Termos e Condições de Uso, o descarregamento de títulos de acesso restrito requer uma licença válida de autorização devendo o utilizador aceder ao(s) documento(s) a partir de um endereço de IP da instituição detentora da supramencionada licença.

Ao utilizador é apenas permitido o descarregamento para uso pessoal, pelo que o emprego do(s) título(s) descarregado(s) para outro fim, designadamente comercial, carece de autorização do respetivo autor ou editor da obra.

Na medida em que todas as obras da UC Digitalis se encontram protegidas pelo Código do Direito de Autor e Direitos Conexos e demais legislação aplicável, toda a cópia, parcial ou total, deste documento, nos casos em que é legalmente admitida, deverá conter ou fazer-se acompanhar por este aviso.

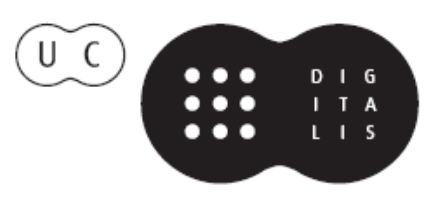




\section{Symposion and Philanthropia in Plutarch}

\section{José Ribeiro Ferreira, Delfim Leão Manuel Troster e Paula Barata Dias (eds.)}

IMPRENSA DA UNIVERSIDADE DE COIMBRA 


\title{
INFLUENCIA DE LOS PROGYMNASMATA EN LA COMPOSICIÓN DE LOS symposta de Plutarco: el caso de El Banguete de los Siete Sabios
}

Ana Vicente Sánchez

Universidad de Zaragoza

\begin{abstract}
In the ancient schools of rhetoric some exercises (called progymnasmata) were practiced in order to teach the students how they should write literary compositions. This training had some influence on the composition of the Septem Sapientium Convivium. These "preliminary exercises" are preserved in some textbooks of prose composition and rhetoric. In this case, the earliest collection of exercises has been used: Theon's Progymnasmata Handbook, which probably dates from the first century after Christ. The three progymnasmata analysed in this

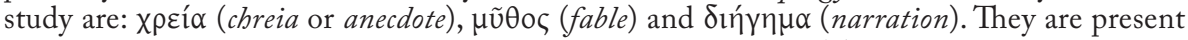
in the Septem Sapientium Convivium in many different ways. The aim of this study is to specify them, to compare their form to Theon's Handbook, and to explain how Plutarch used them.
\end{abstract}

Como reza el título de este trabajo ${ }^{1}$ se va a estudiar la presencia y uso de algunos ejercicios preparatorios propios de las escuelas de retórica en El banquete de los Siete Sabios. Evidentemente nos encontramos ante una composición literaria que, como tal, no va a reproducir con exactitud esos $\pi \rho \circ \gamma \cup \mu v \alpha ́ \sigma \mu \alpha \tau \alpha$, pero sí pueden vislumbrarse las huellas de la formación retórica: unas características y unos rasgos que inequívocamente nos remiten a ellos ${ }^{2}$. Dada la extensión de este trabajo, se va a limitar el análisis

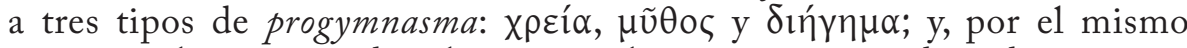
motivo, sólo se expondrá algún ejemplo representativo de cada ejercicio. El punto de partida de estas comparaciones va a ser el manual de Teón, el primero que se nos ha conservado ${ }^{3}$, probablemente contemporáneo ${ }^{4}$ de Plutarco.

${ }^{1}$ Realizado bajo los auspicios del Proyecto de Investigación Hum 2007-64772 de la Dirección General de Enseñanza Superior e Investigación Científica.

${ }^{2}$ Vide otros trabajos que desarrollan esta relación entre los progymnasmata y la técnica compositiva de Plutarco: M. Bellu, 2005 y 2005b; J. A. Fernandez Delgado, 2000 y 2005; L. Miguélez Cavero, 2005; F. Pordomingo Pardo, 2005; A. Vicente Sánchez, 2005.

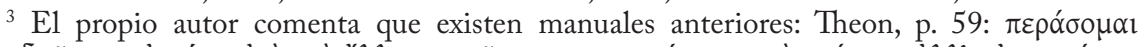

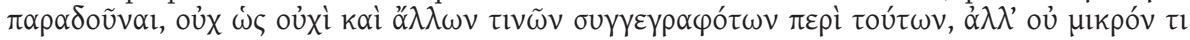

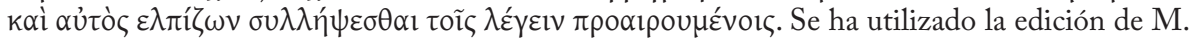
Patillon, 1997, conservando la numeración de la canónica de L. Spengel, Rhetores Graeci, vol. II, Leipzig, 1854.

${ }^{4}$ Compuesto quizá este manual de ejercicios en el siglo I o entre finales del siglo I y principios del II; cf. R. F. Носк \& E. N. O’Neil, 1986, p. 10; M. Patillon, 1997, pp. VII sqq., G. A. Kennedy, 1972, pp. 615 s., y 1983, pp. 54 sqq.y 2003, p. XII s. y 1. Para una datación más tardía, vide M. Heath, 2000, pp. 129 sqq., aunque la crítica en general - y también este trabajo - prefiere esa datación en torno al siglo I. 


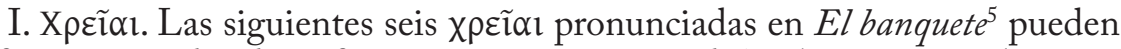
conformar una idea de su forma y uso en este tratado ${ }^{6}$, si bien son muchas más las que contiene la obra ${ }^{7}$.

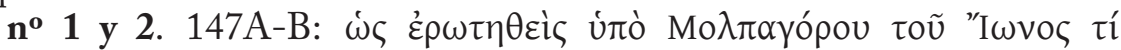

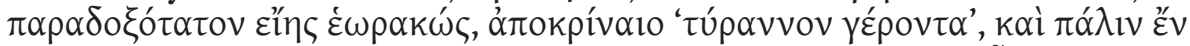

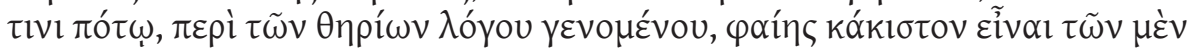

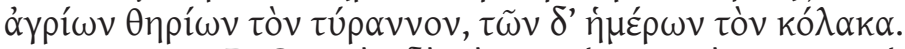

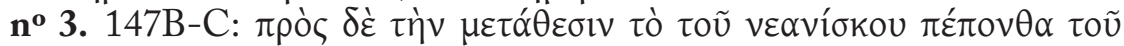

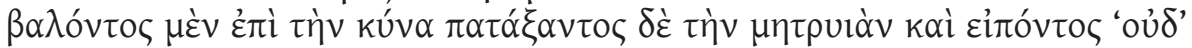

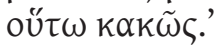

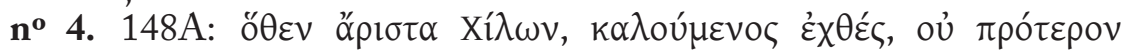

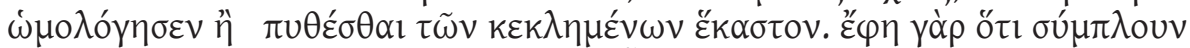

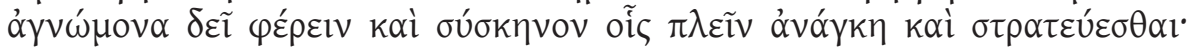

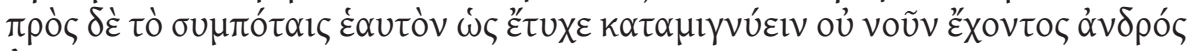
$\dot{\varepsilon} \sigma \tau \mathrm{\tau}$.

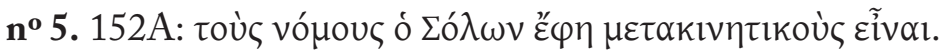

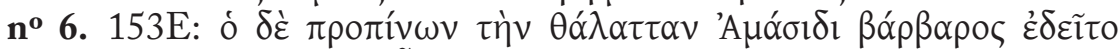

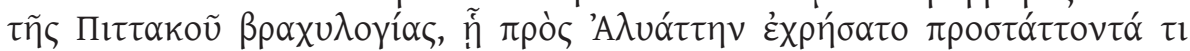

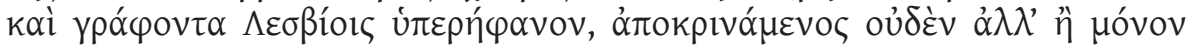

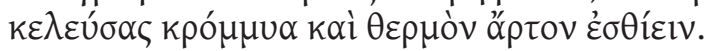

La chreia es, según el manual de Teón, un breve dicho o acción atribuidos a un personaje generalmente conocido $^{8}$, como es el caso de la mayoría que 1985.

${ }^{5}$ El texto procede de la edición de J. Defradas et al., Plutarque. Oeuvres morales, II, París,

${ }^{6}$ Sobre su presencia en De tuenda sanitate praecepta, vide M. Bellu, 2005, pp. 209 sqq. y 2005b, pp. 176 sqq.; sobre su uso en otras obras, vide M. BELLU, 2005b, pp. 325 sqq. (agradezco a la autora, Mariangela Bellu, su gentileza al permitirme consultar su tesis doctoral).

${ }^{7}$ Además de las estudiadas en este trabajo, pueden citarse las siguientes $\chi \rho \varepsilon \tilde{\imath} \alpha$ ı pronunciadas por los personajes: 147C (Tales se sirve de una afirmación de Pítaco), 149A-B (Tales reprocha a Alexídemo mediante las palabras de un lacedemonio anónimo), $155 \mathrm{D}$ (Quilón sustenta su parecer a través de una contestación de Licurgo), 158C (Cleodoro cita una opinión de Tales), 160A-B (Solón utiliza unos versos homéricos). A éstas podrían añadirse las $\chi \rho \varepsilon \tilde{\imath} \propto l$ que Plutarco, a través de la narración de Diocles, se permite utilizar: 147A (una afirmación de Nilóxeno),

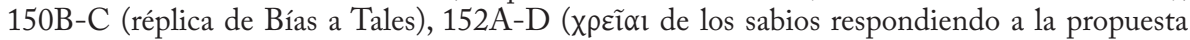
de Periandro sobre reyes y tiranos; a ellas se añade la aportación de Periandro a petición de sus comensales), 152D (intervenciones de Quilón y Esopo), 154D-E (opiniones de los sabios y de Periandro sobre el gobierno igualitario, a petición de Mnesífilo), 155C-D (opiniones de los sabios acerca de la mejor casa, a petición de Diocles), 157B (Cleobulo, para contestar a una demanda de Quersias, cuenta una fábula cuyo "enlace" - vide infra - consiste en una X $\rho \varepsilon i ́ \alpha), 157$ C (primera parte de la contestación de Cleobulo a Cleodoro), 157D (intervención de Tales sobre la persona de Epiménides).

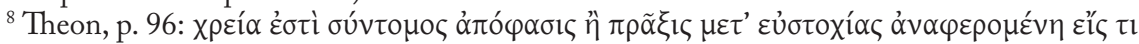

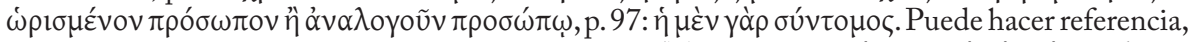

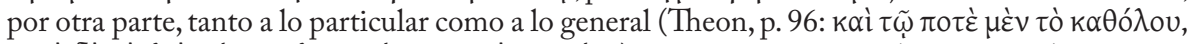

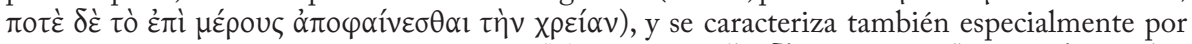

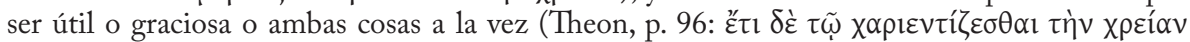

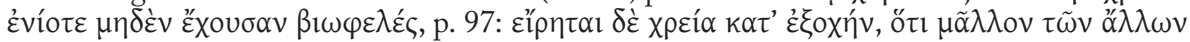

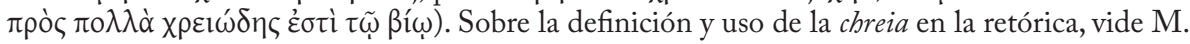


incluye Plutarco en El banquete de los Siete Sabios; las atribuidas a personajes no determinados son muy poco frecuentes, como la del joven de la $\chi \rho \varepsilon \varepsilon^{\alpha} \alpha n^{\circ} 3^{9}$.

Respecto de las clases de chreia, tenemos representados en El Banquete de los Siete Sabios casi todas las que Teón propone: verbales, de acción y mixtas. Así, siguiendo su clasificación, tiene el opúsculo chreiai verbales ${ }^{10}$ - las que sólo contienen un dicho - tanto enunciativas de tipo espontáneo ${ }^{11}$ y de tipo circunstancial (así las chreiai no 2 y 5), como del género llamado "de respuesta", de cuyos tipos utiliza especialmente Plutarco el de indagación y el interrogativocausal: uno consiste no sólo en afirmar o negar, sino en una respuesta extensa ${ }^{12}$ $\left(n^{\circ} 1\right)$ y el otro en añadir a la respuesta una causa, un consejo o algo similar ${ }^{13}$ $\left(n^{\circ} 6\right)^{14}$. Asimismo habla Teón de un último tipo dentro de las verbales que serían las dobles ${ }^{15}$, por contener afirmaciones de dos personajes ${ }^{16}$, aunque una de ellas sola ya puede considerarse una chreia. Otro género de chreiai son las acciones que sin palabras muestran un pensamiento ${ }^{17}$. Y, por último, habla Teón de chreiai mixtas ${ }^{18}$, mezcla de las anteriores y ejemplificadas en no 3 y 4.

Por otra parte las $\chi \rho \varepsilon \tilde{\alpha} \alpha 1$ tienen distintos modos de enunciación según

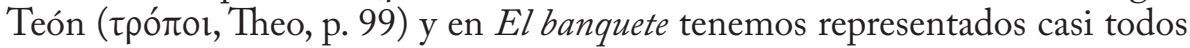
esos modos, de los que aquí vamos a reflejar algunos mediante los seis ejemplos seleccionados. Un modo de enunciación ciertamente frecuente es la sentencia ( $\gamma v \omega \mu \mathrm{o} \lambda$ oүı $\tilde{\omega} \varsigma$, Theon, p. 99), como puede apreciarse en la afirmación de Solón acerca de las leyes ( ${ }^{\circ}$ 5). Igualmente reiterada es la expresión de una

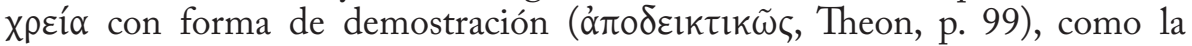
de Quilón respecto de los compañeros de banquete $\left(n^{\circ} 4\right)$. Muy apreciadas

BELLu, 2005b, pp. 65 sqq.

${ }^{9}$ Anónima es también la pronunciada por un lacedemonio en 149A-B.

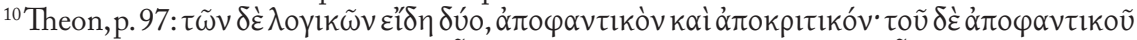

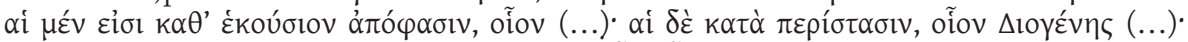

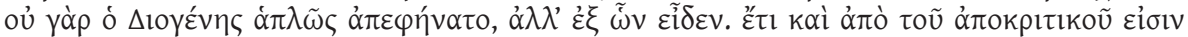

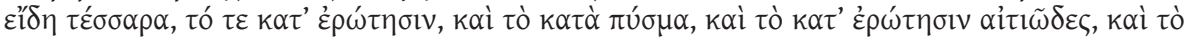

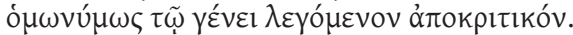

${ }^{11}$ Como la de Pítaco en $147 \mathrm{C}$, o la de Tales en $158 \mathrm{C}$.

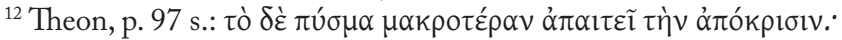

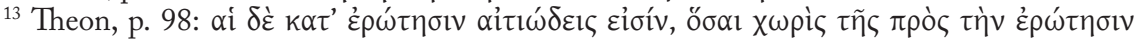

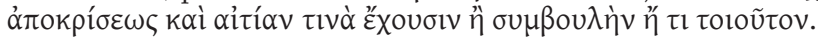

${ }^{14}$ Otros ejemplos son las opiniones de los sabios y de Periandro sobre el gobierno de reyes y tiranos en 152A-D, la chreia de 157B, las opiniones sobre el gobierno igualitario de 154D-E y sobre el de una casa $155 \mathrm{C}-\mathrm{D}$, opiniones todas ellas que responden a algún comensal que así lo ha pedido (a petición de Periandro en 151E-F, de Quersias en 157A, de Mnesífilo en 154C-D y de Diocles en 154F, respectivamente).

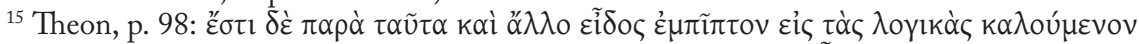

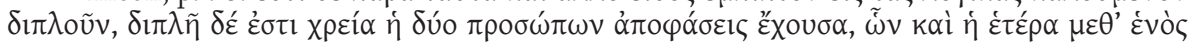

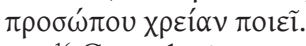

${ }^{16}$ Como las intervenciones de Quilón y Esopo en 152D.

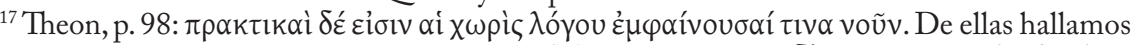

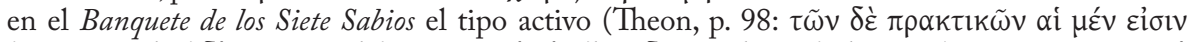

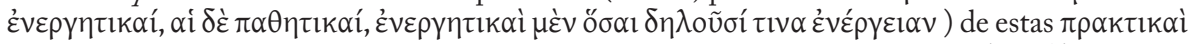
$\chi \rho \varepsilon \tilde{a} \alpha$ en la afirmación de Nilóxeno acerca de la relación de Bías con los reyes (147A).

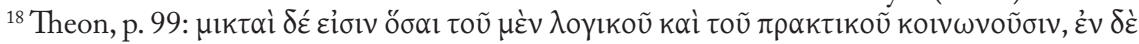

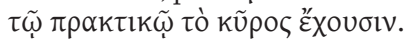




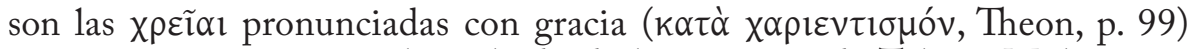
y en El banquete ese es el resultado de la respuesta de Tales a Molpágoras acerca de lo más extraordinario que ha visto: "un tirano viejo" (no 1$)^{19}$. La enunciación puede hacerse de forma figurada ( $\tau \rho \circ \pi \_\tilde{\omega} \varsigma$, Theon, p. 100), y así se expresa Tales al identificar a los tiranos con la peor de las fieras salvajes, y a los aduladores, con las domésticas $\left(\mathrm{n}^{\circ} 2\right)$. Cuando se emite una respuesta distinta a la pregunta realizada, dice Teón que se trata del modo denominado metalepsis ${ }^{20}$, algo que utiliza sabiamente Pítaco para replicar a Aliates cuando éste de un modo soberbio escribió y envió ciertas órdenes a los lesbios, y Pítaco no le contestó otra cosa que animarle a comer cebollas y pan caliente $\left(n^{\circ} 6\right)$. Por último hay un modo que baraja los anteriores, con la posibilidad de múltiples combinaciones ${ }^{21}$, y así, por medio del ejemplo ( $\kappa \alpha \tau \dot{\alpha} \pi \alpha \rho \alpha ́ \delta \varepsilon \imath \gamma \mu \alpha$,

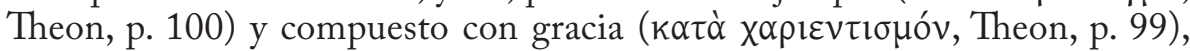
menciona Tales la $x \rho \varepsilon i ́ \alpha$ de un joven ( ${ }^{\circ} 3$ ): "Y en relación con esta situación, he experimentado lo del joven que tirando a su perra, le dio a su suegra y dijo: "no está mal así tampoco"22.

A la hora de componer las chreiai pueden ejercitarse una serie de pasos

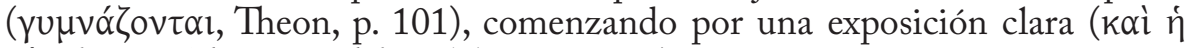

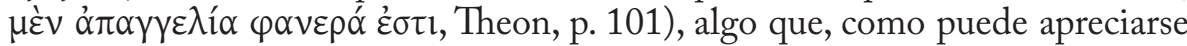
en la selección de textos, Plutarco cumple a la perfección. En segundo lugar, como es natural en un ejercicio escolar, se recomienda practicar los distintos números y $\operatorname{casos}^{23}$ en cada chreia, y también Plutarco ilustrará esta práctica: así pasa en la enunciación del singular al plural en nuestros ejemplos $n^{\circ} 2$, 4 y $5^{24}$, o manteniendo el singular en $n^{\circ} 1,3$ y 6 . En cuanto a los casos, tal y como lo indica Teón ${ }^{25}$, el nominativo no presenta ninguna dificultad y todas

${ }^{19} \mathrm{Si}$ bien a continuación Tales atribuye esta $\chi \rho \varepsilon i ́ \alpha$ a Pítaco, mencionando, además, que la

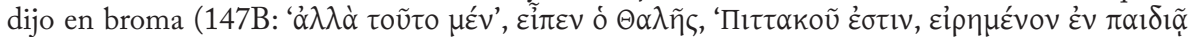

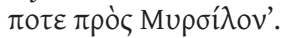

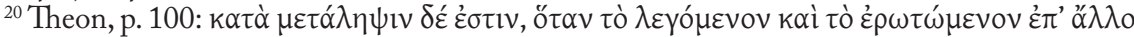

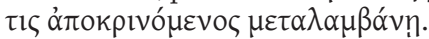

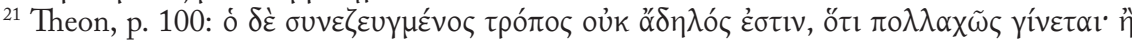

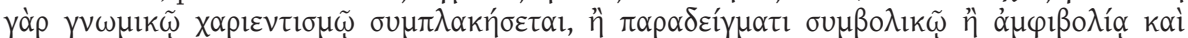

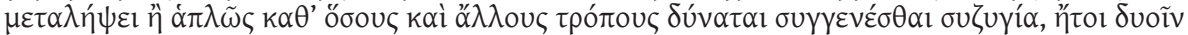

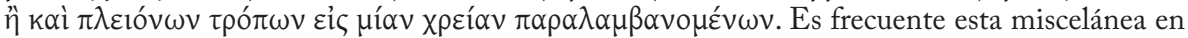
el Banquete: con gracia y de forma alegórica ( $\sigma u \mu \beta 0 \lambda \iota \kappa \tilde{\omega} \varsigma$, Theon, p. 100 - según el uso que Teón le da a este modo $\sigma u \mu \beta 0 \lambda ı \kappa \tilde{\omega}$; vide M. Patillon, p. 23, n. 142 y D. Eloc. 99 sqq.) le replica Bías a Tales acerca de su capacidad de discusión bajo los efectos de Dioniso (150B-C); sentencia más demostración encontramos en la chreia de Cleobulo en 157B; o sentencia más ejemplo en $157 \mathrm{C}$.

${ }^{22}$ La misma combinación aparece en las palabras del lacedemonio anónimo de 149A-B.

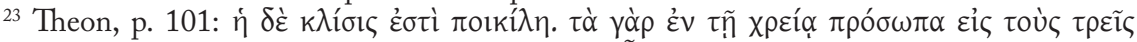

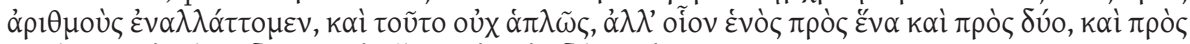

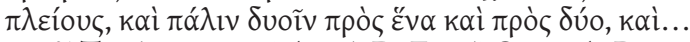

${ }^{24}$ También en 147A, 154D-E, 157C, 160A-B, y en 152A-D y 155C-D combina ese paso con chreiai de singular a singular, que también tiene lugar en 147C, 149A-B, 150B-C, 152D, $155 \mathrm{D}, 157 \mathrm{~B}, 157 \mathrm{D}$ y $158 \mathrm{C}$.

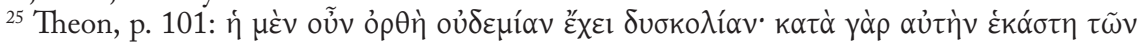

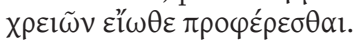


las chreiai suelen enunciarse con dicho caso, como ocurre con la mayoría que utiliza Plutarco $\left(n^{\circ} 4\right.$ y 5 de la selección ${ }^{26}$ ), aunque también incluye alguna chreia enunciada en vocativo ( $\kappa \lambda \eta \tau \iota k \eta ́$, Theon, p. 102 s.; $\mathrm{n}^{\circ} 1$ y 2), genitivo ( $ү \varepsilon \vee ı k \eta े ~ \pi \tau \tilde{\omega} \sigma \iota \varsigma$, Theon, p. 101 s.; no 3 y 6 ) y acusativo ${ }^{27}$. A continuación puede añadirse un epifonema que demuestre de modo apropiado y breve que la chreia es verdadera, hermosa, útil, de acuerdo con la opinión de hombres reputados ${ }^{28}$, como en la $n^{\circ} 3$, que se corrobora mediante otros personajes - Solón y Pítaco -, dignos de mención por compartir el mismo punto de vista ${ }^{29}$; y puede replicarse a partir de los contrarios ${ }^{30}$ : tras las $\chi \rho \varepsilon \tilde{\imath} \alpha 1$ no 1 y 2, como epifonema de réplica, se critica lo poco agradable de la actitud de Tales ${ }^{31}$; en la $n^{\circ} 5$ se considera la imposibilidad de tal afirmación tildándola de "ridícula" ${ }^{2}$. En quinto lugar puede ampliarse o abreviarse la chreia (Theon, p. 103), siendo esta última opción la preferida de Plutarco, parco en casi todas ellas ${ }^{33}$. Y, por último, se pueden refutar las chreiai desde diversos puntos de vista (Theon, p. 104 s.), aunque no todos son siempre posibles. Esta parte del desarrollo de la chreia, dada la brevedad hacia la que tiende Plutarco, no es muy frecuente en El banquete, pero aun así encontramos algún ejemplo como el de la inconveniencia ( $\dot{k} k$

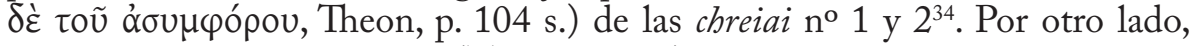
también pueden confirmarse (Theon, p. 105) a partir del argumento contrario

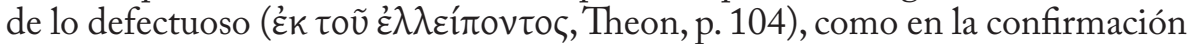

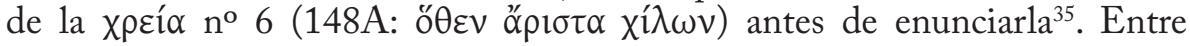
otros elementos amplificadores de la chreia, propone Teón también añadir un proemio (Theon, p. 105 s.), pero dado que Plutarco emplea las chreiai como un elemento más en su narración, evidentemente ha de prescindir de incluir todas las partes de que puede constar según el manual de ejercicios retóricos.

${ }^{26}$ También en 147A, 147C, 149A-B, 150B-C, 152A-D, 152D, 154D-E, 155C-D, 155D, $157 \mathrm{~B}, 157 \mathrm{C}, 157 \mathrm{D}, 158 \mathrm{C}$.

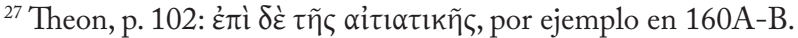

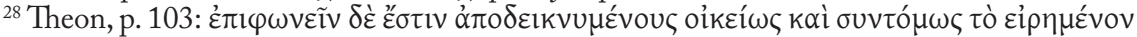

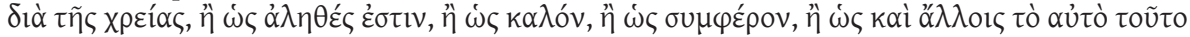

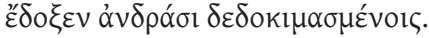

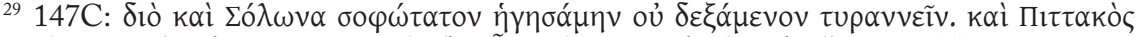

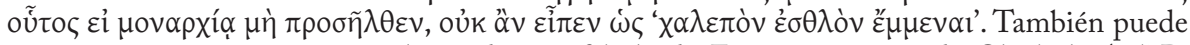
mencionarse como epifonema el uso de una fábula de Esopo por parte de Cleobulo (157B:

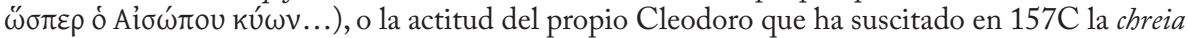
de Cleobulo, quien a continuación la va a comparar con el proceder del médico (157C: kai où

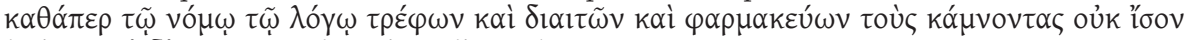

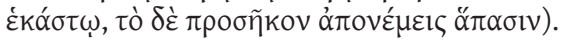

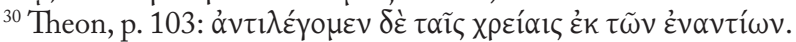

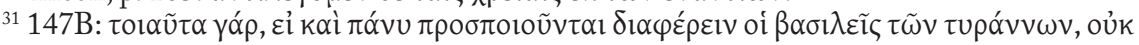
$\varepsilon \dot{u} \mu \varepsilon v \tilde{\omega} \varsigma$ ảkov́ovolv.

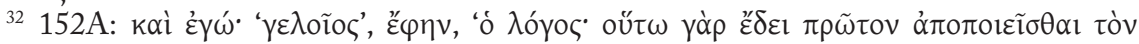

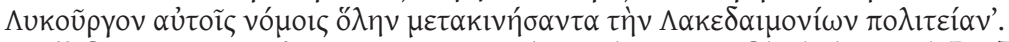

${ }^{33}$ Quizá se extiende un poco más con las explicaciones Cleobulo en 157B y Tales añade los versos de su anfitriona en Éreso para explicar el dicho sobre Epiménides en 157D.

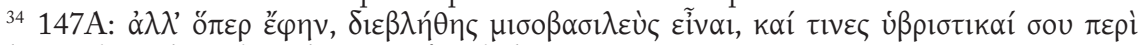

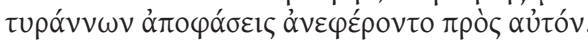

${ }^{35} \mathrm{Y}$ a partir de lo conveniente en 149A-B. 
II. Mũ日ol. A continuación ilustraremos el segundo progymnasma estudiado a través de uno de sus ejemplos ${ }^{36}$, la fábula del lobo y los pastores $(156 \mathrm{~A})^{37}$. Según el progymnasma Пepi $\mu u ́ \theta$ ov es la fábula una composición falsa pero que representa la verdad ${ }^{38}$ y que a pesar de ser falsa e imposible, sin embargo, es verosímil y útili ${ }^{19}$. Puesto que Plutarco las emplea como un elemento más dentro de su composición, no tendremos las fábulas como tal ejercicio retórico con todas sus partes y secciones ${ }^{40}$, pero aun así pueden observarse algunas de las características que propugna la progymnasmatica, como su exposición, su declinación, su enlace a un relato, su abreviación y, en ocasiones, su confirmación. La exposición ( $\alpha \pi \alpha \gamma \gamma \varepsilon \lambda i ́ \alpha$, Theon, p. 74) debe hacerse de una forma sencilla y natural $y$, a ser posible, sin ornato ${ }^{41}$, tal y como nos la presenta Plutarco (156A):

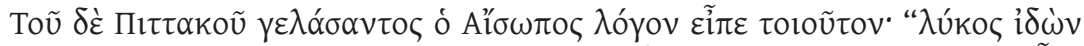

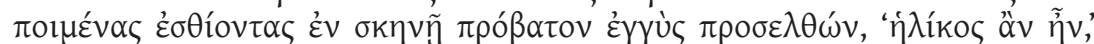

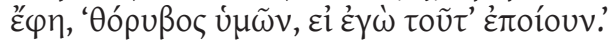

El caso empleado en su declinación ( $k \lambda i ́ v o \mu \varepsilon v$, Theon, p. 74) es el nominativo únicamente $(\lambda \text { úkoৎ... })^{42}$ y pasa de singular a plural. Por otra parte se inserta la fábula $\left(\sigma \cup \mu \pi \lambda \lambda_{\varepsilon} \text { ko } \mu \varepsilon v \text {, Theon, p. } 75\right)^{43}$ tras unos comentarios sobre beber vino (155D-156A) y tras plantearse la cuestión de por qué no bebía vino en ese momento Solón. Anacarsis explica que en realidad teme una ley de Pítaco que sin embargo él se atrevió a transgredir, momento que aprovecha Esopo para contar una graciosa fábula. A continuación le responde de nuevo

${ }^{36}$ Otras fábulas que aparecen en El banquete son: 150A-B, 155B-C, 157A-B, 157B.

${ }^{37}$ Sobre la presencia de la fábula esópica en la obra de Plutarco vide C. GARcía GuAL, 1994, pp. 605 sqq., y sobre la influencia de la preceptiva escolar, vide J.A. Fernández Delgado, 2005, pp. 77 s.; acerca de la fábula y del papel de los animales en el Banquete de los siete sabios, vide M. A. Durán Lopez, 2005, pp. 112 sqq.

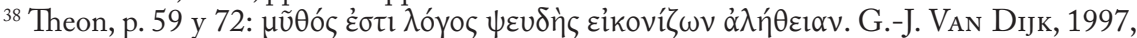
pp. 47 sqq., analiza la información que acerca de la fábula proporciona Teón, cuya definición es la más antigua conservada.

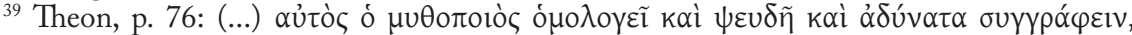

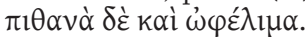

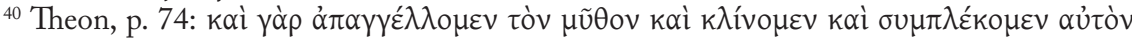

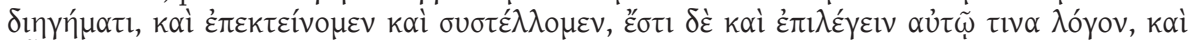

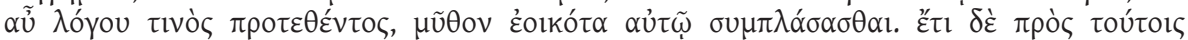
ơv $\alpha \sigma \kappa \varepsilon \cup \alpha ́ \zeta$

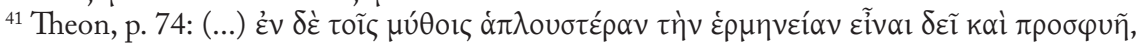

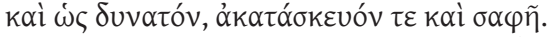

${ }^{42}$ Como ocurre en las fábulas de 150A-B, 155B-C; en 157A-B se declina en acusativo; y en 157B pasa de nominativo a acusativo.

${ }^{43}$ De modo que se enlaza esta fábula a su relato a continuación de éste, como en 157A-B, mientras que otros ejemplos del Banquete realizan un enlace tanto a lo anterior como a lo posterior. Así en 150A-B Esopo cuenta una fábula referida a la actuación de Alexídemo (148E-F, asunto mencionado de nuevo a partir de $149 \mathrm{~F}$ ) - que abandona airado el banquete al considerar que se le había adjudicado una posición deshonrosa -, pero Plutarco hace que sirva igualmente respecto

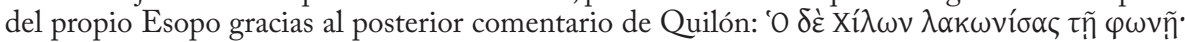

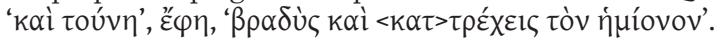




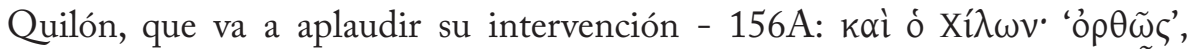

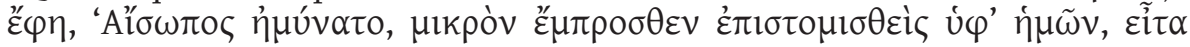

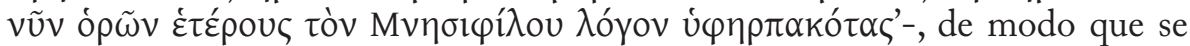
convierte en la confirmación ( $\alpha \alpha \tau \alpha \sigma \kappa \varepsilon v \eta ́$, Theon, p. 76 s.), pues Quilón alaba la conveniencia de la fábula en ese momento. Ante la doble posibilidad de ampliación o abreviación de la fábula (Theon, p. 75) se decanta Plutarco en sus ejemplos de fábula por la abreviación ${ }^{44}$.

III. $\Delta$ ıทүท́ $\mu \alpha \tau \alpha$. Finalmente vamos a ver la puesta en práctica de otro progymnasma igualmente repetido en El banquete de los Siete Sabios ${ }^{45}$, el del relato. Y nos va a servir de ejemplo el primero que Diocles refiere, el relato de Gorgo y el citaredo Arión (160D - 161B y 162A - 162B). Según la definición del manual, un relato debe tratar hechos acaecidos o como si hubieran acaecido $^{46}$, y Plutarco se las ingenia para conseguir estos efectos, si bien en este análisis únicamente se va a hacer referencia a las dos partes clave en el ejercicio

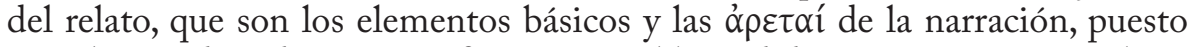
que el resto de indicaciones ofrecen cierta libertad de seguimiento y más bien consisten en una clasificación de las posibilidades que un relato presenta.

Diocles nos informa a la entrada de Gorgo en el banquete de lo siguiente: quién es, cuándo llega, adónde había sido enviado, el motivo, el hecho y la forma de llevarlo a cabo ${ }^{47}$; mientras que a través de las palabras de Gorgo nos enteraremos, además, del momento y del modo de la acción. De esta manera quedan cubiertos los elementos necesarios para constituir un relato, como bien especifica Teón ${ }^{48}$ : el personaje es Gorgo al mando de una expedición religiosa; el asunto es llevar a cabo una $\theta \varepsilon \omega \rho i ́ \alpha$ y sacrificios a Posidón; la acción tiene lugar en el Ténaro; la expedición religiosa había sido motivada por unos oráculos; el momento de la acción es durante la noche del último día de los tres que duró la expedición; y, por último, según las indicaciones de Teón ${ }^{49}$, podemos decir que el modo de la acción es involuntario por azar, ya que ellos no esperaban encontrarse lo que sucedió en la playa (160F sqq.), o por necesidad, puesto que son unos oráculos divinos los que han inspirado la expedición junto al mar ${ }^{50}$. Por lo tanto ya se han expuesto los elementos necesarios para que el relato sea completo, siguiendo las instrucciones de Teón ${ }^{51}$.

${ }^{44}$ Parece ser esta una tendencia general en el uso de la fábula por Plutarco, vide J. A. Fernández Delgado, 2005, p. 80 s.

${ }^{45} \mathrm{Y}$ en otras partes de la obra plutarquea, vide F. Pordomingo Pardo, 2005, p. 130.

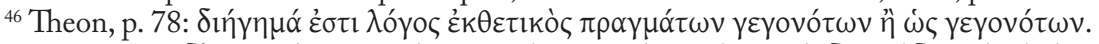

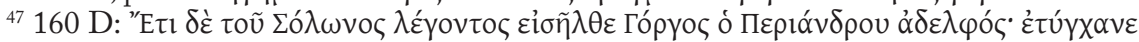

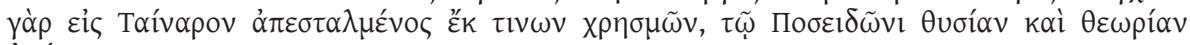

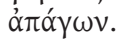

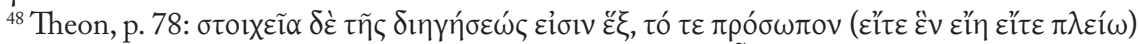

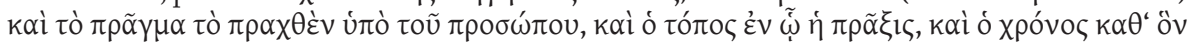

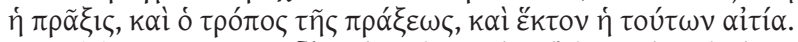

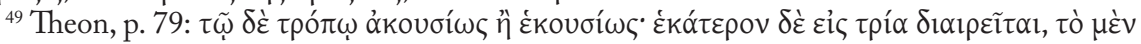

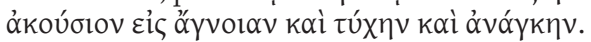

${ }^{50}$ Después comenta Gorgo que la divinidad parece estar detrás de todo el asunto (162B:

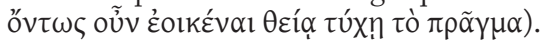

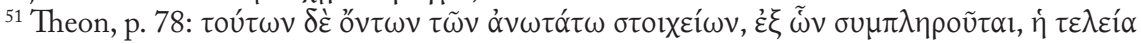


Y respecto de la segunda de las claves que mencionábamos, las ỏ $\rho \varepsilon \tau \alpha i ̀ ~$ $\delta ı \eta ү \tilde{\sigma \varepsilon \omega \varsigma, ~ e n ~ e l l a s ~ s e ~ b a s a ~ l a ~ e f i c a c i a ~ d e l ~ r e l a t o ~ y, ~ e n ~ l a ~ m e d i d a ~ d e ~ l o ~ p o s i b l e, ~}$ deben estar presentes en toda narración: claridad, concisión y verosimilitud ${ }^{52}$. Siguiendo las indicaciones de Teón, puede decirse que este relato de El banquete se ha compuesto con claridad en cuanto al tema y al estilo (Theon, p. 80): por una parte en cuanto al tema ${ }^{53}$, puesto que ni siquiera la actuación de los delfines resultaría ajena al auditorio ${ }^{54}$ y solamente se narra un hecho ${ }^{55}$, sin mezclarlo con otros simultáneos, además de hacerlo con $\operatorname{orden}^{56}$ y sin digresiones ${ }^{57}$; por otra parte, en cuanto al estilo ${ }^{58}$, deben evitarse las nuevas creaciones y los términos poéticos, los metafóricos, arcaicos, extranjeros y homónimos, la ambigüedad en la expresión, el hipérbaton (suave se permite), las digresiones extensas, la falta de nombres o utilizar un caso que puede hacer referencia a dos nombres distintos dando lugar a confusión: la única excepción que aparece en

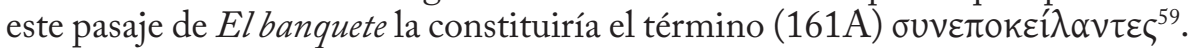
También la concisión va a surgir de los hechos y del estilo. Lo primero ${ }^{60}$ se consigue cuando no se incluyen muchos asuntos, no se insertan unos en otros, se dejan de lado los que se sobreentienden, no se empiezan los asuntos desde lejos, etc. Y para ser conciso en cuanto al estilo hay que evitar sinónimos, perífrasis, elementos superfluos, compuestos, y decantarse por los nombres más

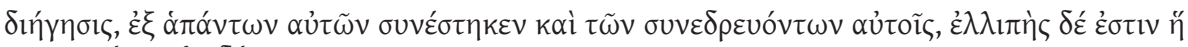

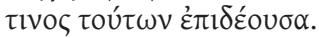

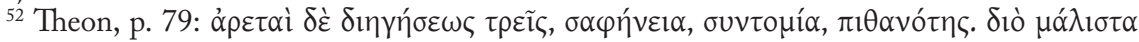

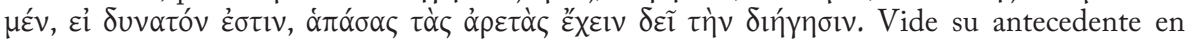
Anaximen. Rh. 1438 a 3-1438 b 13.

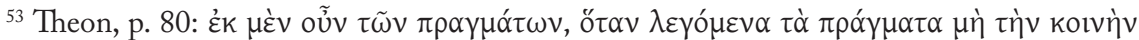

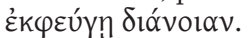

${ }^{54}$ Son animales de frecuente aparición, además de en otros ámbitos artísticos, en la literatura griega, como en Ilíada 21.22 sqq. (se emplea el delfín para comparar el temor que provoca en los peces pequeños a los que sin duda va a devorar, con la actitud de los troyanos en el río Janto huyendo de un Aquiles enfurecido); famosos por su rapidez, como señala Píndaro en Nemea VI 64; conocidos por sus danzas marinas (Eurípides, Helena, v. 1455); y son numerosas las fábulas esópicas con delfines, como la 62, 73, 113 o la 145 (edición de B. E. Perry, Aesopica, I, Urbana, 1952).

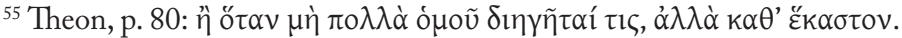

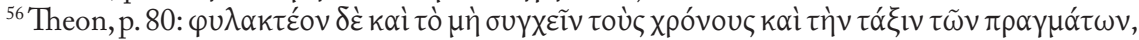

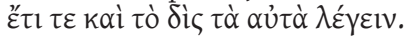

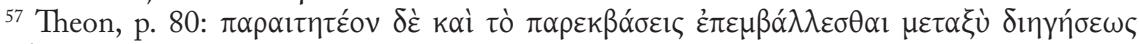

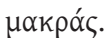

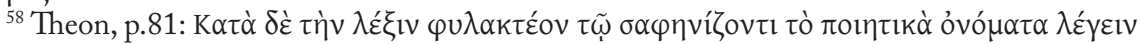

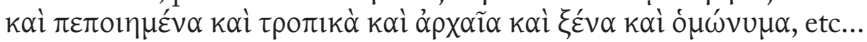

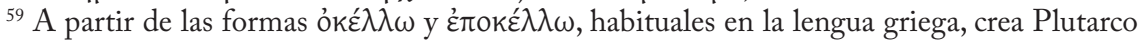

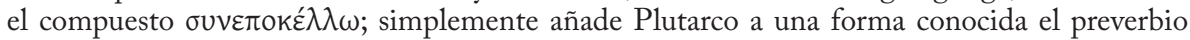
$\sigma u v$ - para reforzar la imagen de que los delfines realizaban el depósito de Arión conjuntamente y todos a la vez sobre la playa (véase también el uso inmediatamente anterior de $\sigma \cup v \alpha \gamma \alpha \gamma o ́ v \tau \varepsilon \varsigma$, 161A).

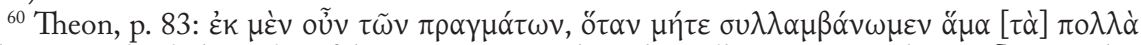

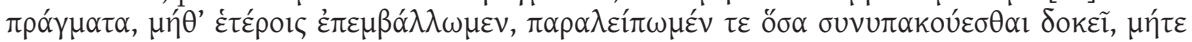

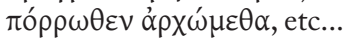


breves, sin llegar a caer ni en la vulgaridad ni en la oscuridad ${ }^{61}$. Todos estos requisitos los cumple Plutarco con cierta seriedad en su relato. Por último,

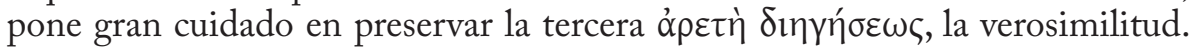
La conversación privada de Gorgo con Periandro llama la atención de nuestro narrador Diocles por las reacciones que en el tirano provoca. Tanto es así que Periandro termina por dirigirse alegremente a los comensales para hacerles partícipes de la historia que acaba de oír. Pero antes se cuestiona el tirano la conveniencia de hacerlo público dado que no cree que parezca verdadero y

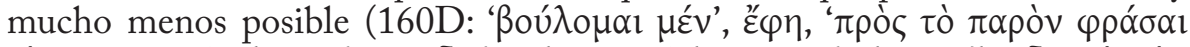

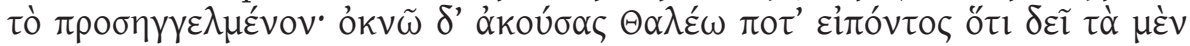

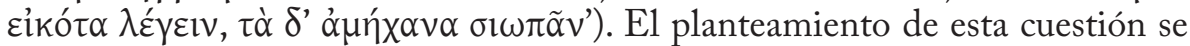
debe probablemente a que, tal y como se especifica en los manuales escolares, un relato debe tratar hechos acaecidos o como si hubieran acaecido, según la definición de Teón que citábamos al principio. La verosimilitud es, además, uno de los medios para realizar la confirmación de un relato (Theon, p. 93 s.), que es parte importante en el desarrollo de cualquier progymnasma (Theon, p. 65), y el narrador, evidentemente, tratará de reforzar esa credibilidad al máximo ${ }^{62}$. Por ello, la mayor preocupación de Plutarco antes de pasar a la narración, es sustentar de alguna manera esa credibilidad de cara a los comensales y a los lectores. Pero Bías va a echarle un cable a Periandro igualmente a través de otra máxima también de Tales, con la intención de no dar por sentada la

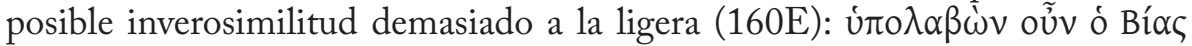

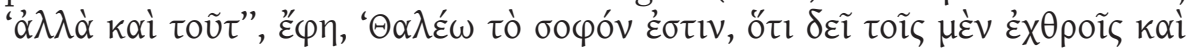

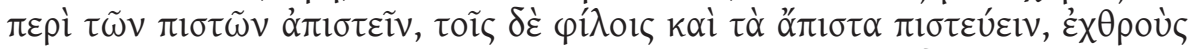

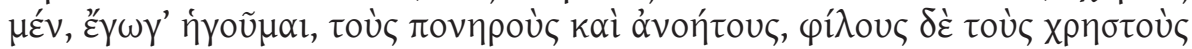

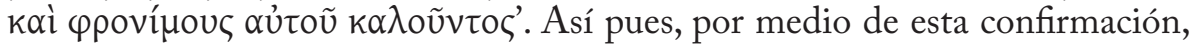
queda un tanto salvaguardada la verosimilitud del relato y Periandro puede ya ceder la palabra a su hermano Gorgo.

En este trabajo se han utilizado tres ( $\chi \rho \varepsilon i ́ \alpha, \mu \tilde{v} \theta$ o y $\delta ı \eta ́ \gamma \eta \mu \alpha)$ de los distintos ejercicios que se practicaban en las escuelas de retórica y que sirven, según las indicaciones de Teón, en cualquier tipo de composición (Theon, p. 60 s., 70 s.), cuya corrección depende en gran medida del empleo de estos progymnasmata, de los que proporciona ejemplos presentes en autores consagrados como Heródoto, Tucídides, Platón, Demóstenes, etc. (Theon, p. 66 s.). A través de los casos aquí estudiados se ha podido constatar la coincidencia entre esa preceptiva escolar y algunos aspectos de El banquete de los Siete Sabios, de modo que podemos comprender mejor la técnica compositiva de Plutarco: por una parte, trabaja en buena medida de acuerdo con las teorías retóricas de la época, y, por otra parte,

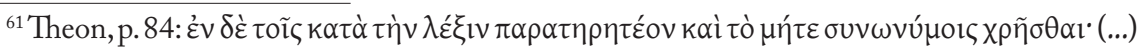

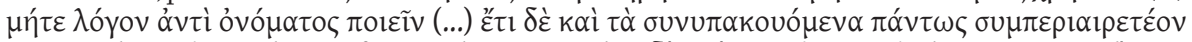

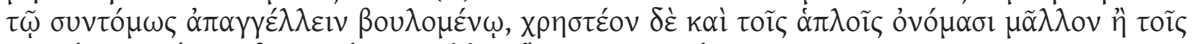

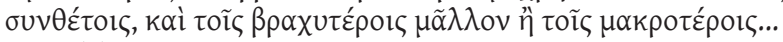

${ }^{62}$ Teón señala que se confirma y refuta un relato a través de los mismos medios que ha explicado para la fábula - a los que puede añadirse algún otro -, y también en ese progymnasma comienza destacando la importancia de la defensa de la verosimilitud para ratificar cada fábula (Theon, p. 76). 
puede observarse que, además de un trasfondo filosófico-moral-religioso, la forma está sumamente cuidada, pues lo contrario sería un grave error, como advierte Teón en las primeras líneas de su manual (cf. Theon, p. 59).

\section{Bibliografía CITAdA}

Bellu, M., "La chreia en el De tuenda sanitate praecepta de Plutarco", in M. Jufresa et AL. (eds.), 2005, pp. 209-16.

La Chreia en los Moralia de Plutarco, Tesis Doctoral, Universidad de Salamanca, 2005B.

Durán López, M. A., "Los animales en las imágenes de Plutarco", in J. Boulogne (ed.), Les Grecs de l'Antiquité et les animaux. Le cas remarquable de Plutarque, Lille, 2005, pp. 111-19

Fernández Delgado, J. A., "Le Grillus, une éthopée parodique", in L. Van Der Stockt (ed.), Rhetorical theory and praxis in Plutarch. Acta of the IV International Congress of the I.P.S. (Leuven, July 3-6), LovainaNamur, 2000, pp. 171-81.

"La fábula en Plutarco: de la historieta ejemplificante al ejercicio progimnasmático", in M. Jufresa ET AL. (eds.), 2005, pp. 77-84.

García Gual, C., "Esopo en Plutarco", in M. García Valdés (ed.), Estudios sobre Plutarco: ideas religiosas. Actas del III Simposio Internacional sobre Plutarco (Oviedo, 1992), Madrid, 1994, pp. 605-14.

Heath, M., "Theon and the history of the Progymnasmata", GRBS, 43 (2000) 129-60.

Hock, R. F. \& O'Neil, E. N., The chreia in ancient rhetoric. vol. I, The Progymnasmata, Atlanta, 1986.

Hunger, H., Die Hochsprachliche profane Literatur der Byzantiner. Erster Band: Philosophie, Rhetorik, Epistolographie, Geschichtsschreibung, Geographie, Múnich, 1978.

Jufresa, M. Et Al. (eds.), Plutarc a la seva època: paideia i societat. Actas del VIII Simposio español sobre Plutarco (Barcelona, 6-8 de Noviembre, 2003), Barcelona, 2005.

Kennedy, G. A., The art of rhetoric in the Roman world, 300 B. C. - A. D. 300, Princeton, 1972.

Greek rhetoric under christian emperors, Princeton, 1983.

Progymnasmata. Greek textbooks of prose composition and rhetoric, Leiden/ Boston, 2003. 
Miguélez Cavero, L., "La teoría de la ekphrasis en Plutarco", in M. Jufresa ET AL. (eds.), 2005, pp. 379-86.

Patillon, M., Progymnasmata. Aelius Théon, París, 1997.

Pordomingo Pardo, F. "Los papeles escolares y Plutarco", in M. Jufresa et AL. (eds.), 2005, pp. 117-32.

Porter, S. E. (ed.), Handbook of classical rhetoric in the hellenistic period 330 BC - AC 400, Leiden/Nueva York/Colonia, 1997.

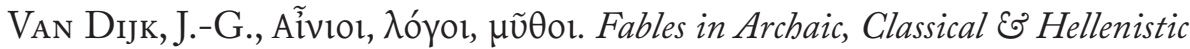
Greek literature. With a study of the theory $\mathcal{E}^{2}$ terminology of the genre, Leiden-Nueva York-Colonia, 1997.

Vicente Sánchez, A., "Plutarco, Sobre si es más útil el agua o el fuego: una tesis progymnasmatica”, in M. Jufresa ET AL. (eds.), 2005, pp. 507-16. 